\title{
Safety Coefficient of the Mechanical Lifeline of Spiders
}

\author{
Shigeyoshi OSAKI ${ }^{\dagger}$ \\ Department of Chemistry, Faculty of Medicine, Nara Medical University, Kashihara, Nara 634-8521, Japan
}

(Received November 26, 2002; Accepted January 27, 2003)

\begin{abstract}
I studied the safety coefficient of the Nephila clavata spider's dragline, which was defined as the ratio of the elastic limit strength of the dragline to the spider's weight, at all stages of the spider's growth. It was found that the dragline gave a safety coefficient of about 3 for young or immature spiders and that of about 2 for mature spiders. It became clear that a high safety coefficient of about 3 could be ascribed to the large cross section area per spider's weight. With respect to the mechanical safety coefficient, the draglines may be overly designed to protect immature spiders surrounded by various dangers while they may be designed to give the most efficient safety for mature spiders. The concept of safety obtained for immature and mature spiders may be applied to the development of a new type of industrial materials and also to animals and humans.
\end{abstract}

KEY WORDS Safety Coefficient / Dragline / Mechanical Lifeline / Spider / Danger / Industrial Material /

Every living thing must be concerned with the problem of its own safety since it is always surrounded with various dangers. Hence, it is important to understand the concept of safety as it relates to preventing animals and humans from having dangerous accidents depending on the living environment which changes with their growth. Such a concept of safety will be useful to macromolecular materials.

The quality of safety may change, depending on the living environment of spiders. A spider secretes silks of draglines, which act as a lifeline when she falls from her web. The draglines are an appropriate object for studying the safety concept from the mechanical point of view, since the total weight of the spider is supported only by the draglines. ${ }^{1,2}$

Many researchers have studied the mechanical properties such as the breaking strength and the elastic modulus of a spider's dragline. ${ }^{3-7}$ Previously, ${ }^{1,2}$ this author found that the elastic limit strength of draglines was about twice the spider's weight and that the dragline gave the most efficient safety as a mechanical lifeline.

However, there have been no reports on the elastic limit strength of draglines for immature spiders because it has been very difficult to prepare the dragline samples with being very thin and mechanically weak out of immature spiders and then to measure the forceelongation curves. Recently, we have overcome the difficulty in preparing the dragline samples out of immature spiders by preventing an extra stress applied to the very thin draglines and modified an apparatus for obtaining stress-strain curves reflecting the very low strength of the draglines with high accuracy., ${ }^{1,2}$ And we determined the elastic modulus of draglines from
Nephila clavata spiders collected at different stages of growth. ${ }^{8}$

It is interesting to examine the quality of safety during the spider's entire life from the viewpoint of growth and evolution because the quality of dangers changes with their growth. If the mechanical properties of draglines are measured at all stages of a spider's growth, it will be possible to understand the concept of the safety for spider's growth and then to give fundamental data for industrial silks produced by using the genetic technology. 9

The present study describes the safety coefficient (SC) of the draglines prepared during the spider's entire life and also the spider's weight per the cross section area of the dragline from the structural point of view. The results may provide a reasonable explanation of the change in the safety coefficient related to the spider's growth and also a useful probe for developing mechanically efficient materials related to the safety.

\section{EXPERIMENTAL}

I carefully sampled very thin draglines out of Nephila clavata spider (Japanese golden web spider) falling naturally from a wooden bar such that an extra force above the elastic limit strength should not be applied to the draglines in order to avoid the mechanical hysteresis of the draglines, as shown in Figure 1 of ref 8 . The draglines act as a lifeline for the mature spiders, which face death when jumping or falling to capture insects and escape rapidly from invaders.

In Japan, $N$. clavata spiders are born from the egg

†To whom correspondence should be addressed (E-mail: s-osaki@naramed-u.ac.jp). 


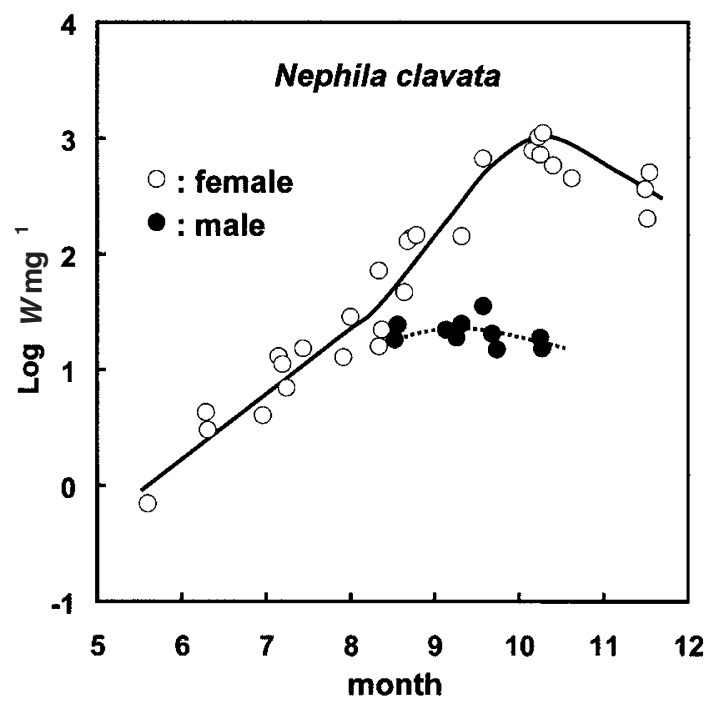

Figure 1. Weight of $N$. clavata (Japanese golden web spider) spiders at different months. N. clavata spiders in Japan are born from the egg cocoon in late spring and reach the divergence of the sex in late summer. Then, male spiders visit the orb-webs of female spiders for the courtship in autumn. After the mating, female spiders produce an egg cocoon in late autumn and soon die. $\bigcirc$ : female, 0 : male.

cocoon in late spring and reach the divergence of the sex, accompanied with the final ecdysis in summer. Male spiders visit the orb-webs of female spiders for courtship in autumn. After mating, pregnant spiders produce egg cocoons in late autumn and soon die. The weight $(W)$ of the $N$. clavata spider in Japan increases exponentially from juvenility in spring to maturity in autumn. For example, a spider's weight increases from about $1 \mathrm{mg}$ in spring, to about $50 \mathrm{mg}$ in summer, and up to about $1 \mathrm{~g}$ in autumn (see Figure 1). It is very difficult to recognize the difference in the spider's sex before late summer. Divergence of the sex into female and male occurs in late summer when the spider's weight reaches about $50 \mathrm{mg}$.

At several stages of spider's life I carefully sampled draglines, ascertained by a scanning electron microscopy (SEM) whether the sample is true draglines and then measured their force-elongation curves.

The average densities of draglines at $20^{\circ} \mathrm{C}$ were determined to be $1.13 \mathrm{~g} \mathrm{~cm}^{-3}$ and $1.29 \mathrm{~g} \mathrm{~cm}^{-3}$ for $N$. clavata in July and October, respectively, by use of the floatation method. ${ }^{10}$

Force-elongation curves of draglines with a length of $4 \mathrm{~cm}$ were carefully measured with high accuracy using a modified instrument manufactured by Instron, Japan with a stretching velocity of $3.3 \times 10^{-4} \mathrm{~m} \mathrm{~s}^{-1}$. The sample draglines were prepared from immature $N$. clavata spiders by preventing an extra stress applied to the very thin draglines. The mechanical measurements were repeated four times for samples, and the data were av-

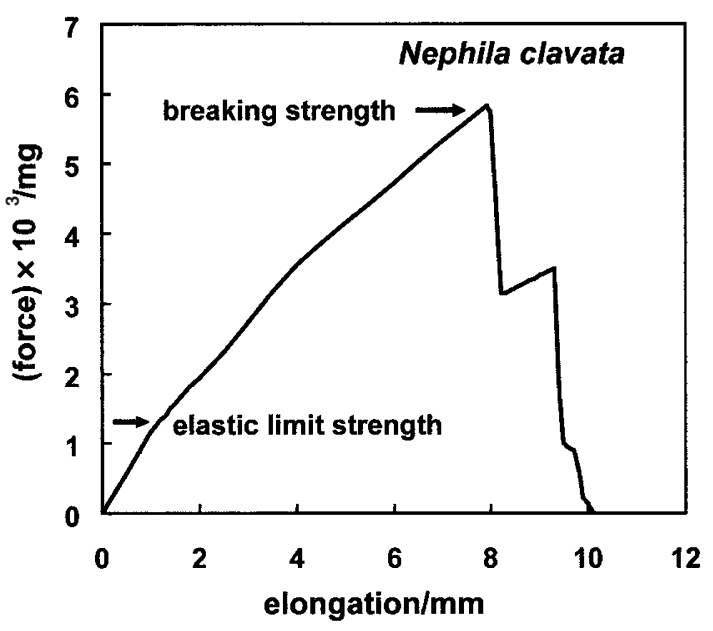

Figure 2. Force-elongation curve obtained for a dragline with a length of $4 \mathrm{~cm}$ secreted from a $N$. clavata spider with a weight of $600 \mathrm{mg}$ at a stretching velocity of $3.3 \times 10^{-4} \mathrm{~m} \mathrm{~s}^{-1}$. Elastic limit strength is defined as the force at the point where the forceelongation behaviour changes from linear to nonlinear and the breaking strength is defined as the force at the breaking point.

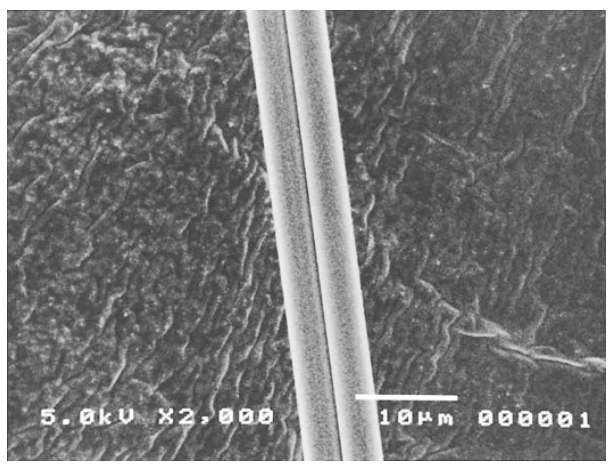

Figure 3. Scanning electron microphotograph of a dragline secreted from a $N$. clavata spider. The electron microphotograph shows that the dragline actually consists of double filaments, each of which has a nearly circular cross section.

eraged. Here, the elastic limit strength determined from the observed force-elongation curves is defined as the force where the force-elongation behaviour changes from linear to nonlinear, ${ }^{1,2,8}$ and the breaking strength is defined as the force at the breaking point, as shown in Figure 2. The errors in the observed values of elastic limit strength and cross section area were 5\%.

Even though the dragline is seen as a single line with the naked eye, an electron microphotograph shows that the dragline actually consists of double filaments (see Figure 3), each of which has a nearly circular cross section. The diameter of each filament was determined with the scanning electron microscope, the area of each cross section was sum up and then the total area $(S)$ of cross section of double filaments was calculated.

\section{RESULTS}

It is necessary to give a standard for evaluating the 


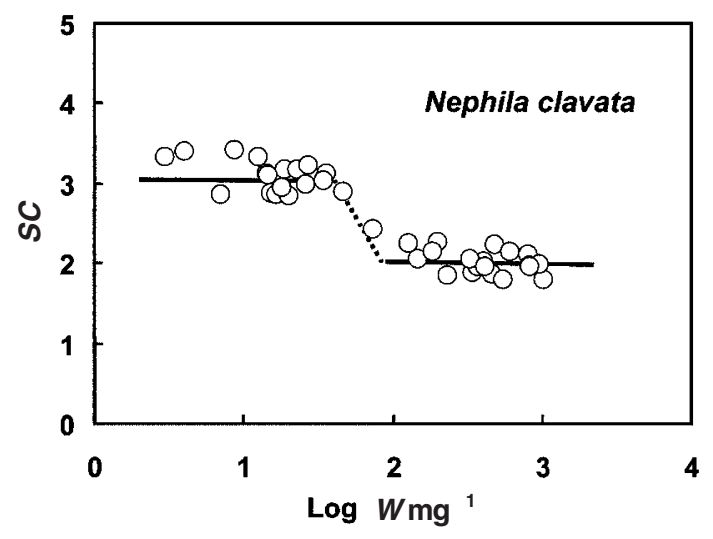

Figure 4. The spider's weight $(W)$ dependence of the safety coefficient $(S C)$ of draglines secreted from $N$. clavata spiders. The $S C$ is defined as the ratio of the elastic limit strength of dragline to the spider's weight, at all stages of the spider's growth. The dragline gives a $S C$ of about 3 for immature spiders of less than $50 \mathrm{mg}$ and that of about 2 for mature spiders of more than $50 \mathrm{mg}$.

degree of safety when the safety is discussed. A variation in the observed values of the elastic limit strength included in the linear region is very small while that of the breaking strength included in the nonlinear region is large. A spider has to entrust her life to the draglines with reliability when her falling and moving. Thus, it is reasonable to accept the elastic limit strength of draglines, whose reliability is obviously higher than that of the breaking strength, for each spider as the standard. Here, the ratio of the elastic limit strength of dragline to the spider's weight is defined as the safety coefficient $(S C)$, which is given as the standard for evaluating the degree of safety. 1,2

Figure 4 shows the safety coefficient $(S C)$ plotted against the spider's weight $(W)$. The $S C$ was determined to be about 2 for mature spiders weighing more than $50 \mathrm{mg}$ and to be about 3 for immature spiders weighing less than $50 \mathrm{mg}$, even though a variation in data was observed. The safety coefficient changes drastically from 3 to 2 around the spider's weight of $50 \mathrm{mg}$. The larger $S C$ means the higher degree of safety since the draglines are mechanically stronger for spider's hanging.

The $S C$ of 1 corresponds to the case that one filament of double filaments constituting the draglines is broken down. And such a value of 1 gives the minimum one which is just able to supports spider's weight because the breaking of the remaining filament will lose spider's life. Even though the larger value of $S C$ gives the higher degree of safety, on the other hand, it needs the high energy for producing the strong draglines. Therefore, the $S C$ of 2 is the most efficient value from the viewpoint of safety and energy.

The safety coefficient is the physical quantity without considering the structure of the draglines. Here, it is

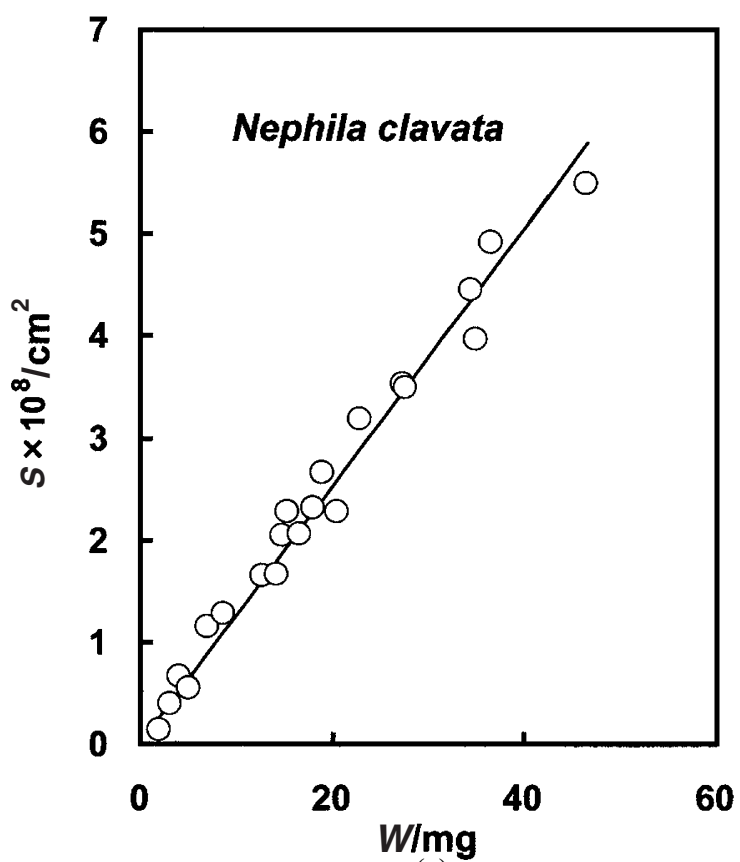

(a)

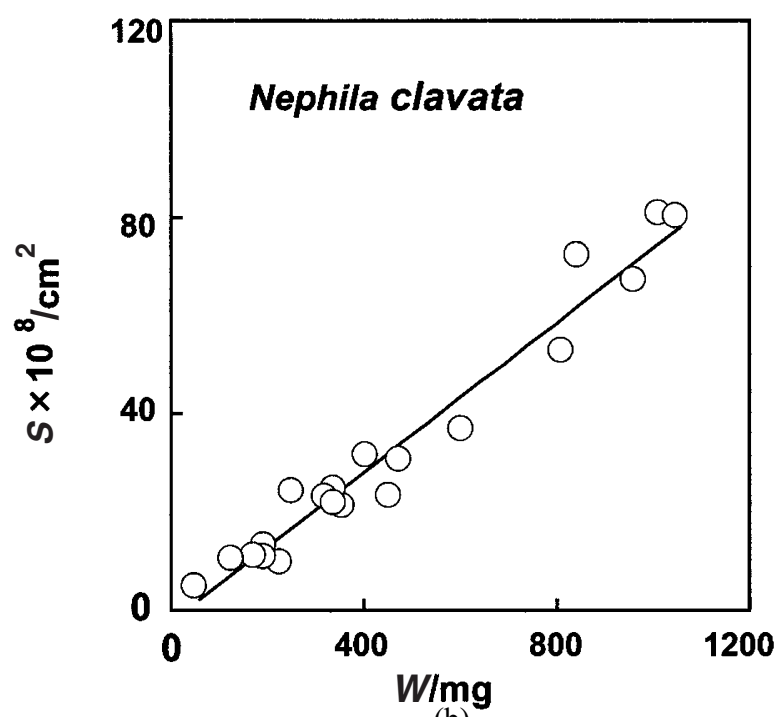

(b)

Figure 5. (a) The cross section area $(S)$ of draglines plotted against the spider's weight $(W)$ for immature spiders ( $N$. clavata) weighing less than $50 \mathrm{mg}$. (b) The $S$ of draglines plotted against the $W$ for mature spiders (N. clavata) weighing more than $50 \mathrm{mg}$.

necessary to consider the draglines from the structural point of view when we examine whether the draglines are an appropriate material for a lifeline. As an actual problem, it is important to estimate the value of the spider's weight per the cross section area of the dragline because the total weight of a spider is applied to the cross section area of the draglines. Such a value will give one standard for evaluating the safety.

Figures 5a and 5b show the cross section area $S$ of a dragline plotted against the spider's weight $(W)$. The $S$ increases with the increasing $W$. We can observe a break at a weight of about $50 \mathrm{mg}$. 
For spiders weighing less than $50 \mathrm{mg}$, the following empirical equation was obtained:

$$
S=1.26 \times 10^{-9} \mathrm{~W}
$$

with a correlation coefficient of 0.974 . And for spiders weighing more than $50 \mathrm{mg}$, the following equation was also obtained:

$$
S=7.60 \times 10^{-10} \mathrm{~W}-2.321 \times 10^{-8}
$$

with a correlation coefficient of 0.957 .

The slope in Figures 5a and 5b corresponds to the cross section area per spider's weight, giving a dimension of $\mathrm{cm}^{2} / \mathrm{mg}$. The slope $(S / W)$ obtained for spiders of less than $50 \mathrm{mg}$ is larger by about $3 / 2$ than that for spiders of more than $50 \mathrm{mg}$. This means that the higher value of $S / W$ for immature babies is safer for spider's hanging so far as the mechanical quality of dragline is almost the same during the spider's entire life. Thus, the $S / W$ gives a parameter for evaluating the safety.

The reciprocal of the slope corresponds to the ratio $(W / S)$, which corresponds to the spider's weight $W$ perfectly applied to the cross section area $\mathrm{S}$ of the dragline when the spider falls down. The $W / S$ reaches about $7.94 \times 10^{8} \mathrm{mg} \mathrm{cm}^{-2}$ (= $78 \mathrm{MPa}$ ) for immature spiders of less than $50 \mathrm{mg}$ and about $1.32 \times 10^{9} \mathrm{mg} \mathrm{cm}^{-2}$ $(=129 \mathrm{MPa})$ for mature spiders of more than $50 \mathrm{mg}$. The $W / S$ for immature spiders was smaller by about $2 / 3$ than that for mature spiders. This means that the cross section area of the draglines per spider's weight is larger for immature spiders than that for mature ones. The draglines of immature spiders may be designed to be safer than that of mature spiders from the structural point of view. Since the spider can hang more safely from a dragline having smaller $W / S$, it is reasonable to use $S / W$ as a parameter for evaluating the safety of a dragline. Thus, such results of this study obtained from the viewpoint of geometrical structure support the safety coefficients as a physical quantity, shown in Figure 4.

\section{DISCUSSION}

It was found that the safety coefficient changed from 3 to 2 with spider's growth. A drastic change in the safety coefficient was observed at about $50 \mathrm{mg}$ of $N$. clavata spiders in summer when the divergence of the sex and the rapid increase in female spider's weight after the final ecdysis occurred. The spider's weight of about $50 \mathrm{mg}$ corresponds to that at the stage that spiders grow from immature to mature in summer. Such a change is supported by the results that the cross section area of draglines per spider's weight and the elastic modulus changed drastically at about $50 \mathrm{mg}$ of the spider's weight. ${ }^{8}$
Immature spiders looking like slender are required to escape rapidly from various invaders and to secrete strong draglines to catch insects as large as possible. Female spiders rapidly grow fat just after the mating. Thus, the relatively thick and mechanically strong draglines may be required for immature spiders to survive without fears of breaking down. If draglines are thick and strong per spider's weight, immature spiders will be able to act safely without fears of breaking down of the draglines. Actually, the spider's weight increases exponentially with month while the cross section area of draglines related to the size of secreting gland increases gradually with month.

Thus, the safety coefficient of about 3 should be based on relatively thick draglines per spider's weight for young or immature spiders while the safety coefficient of about 2 should be most efficient for mature spiders from the viewpoint of energy consumption and safety. It may be the hereditary result of parents having to leave their offspring to survive over the spider's long evolution of 400 million years, closely depending on her genes.

On the other hand, immature humans and animals are much different in a figure from mature ones. The concept on the safety for humans and animals may be related to the results obtained in the present study.

In conclusion, with respect to the mechanical safety coefficient, draglines may be overly designed to protect young or immature spiders surrounded by various dangers without help of their parents while they may be also designed to give the most efficient system of safety for mature spiders. The structural findings will give important information on designing a new type of industrial materials. Furthermore, we should consider the safety based on the various dangers induced between the living things and its environment and such a concept of safety will be applied to young animals and humans.

\section{REFERENCES}

1. S. Osaki, Nature, 348, 419 (1996).

2. S. Osaki, Int. J. Biol. Macromol., 24, 283 (1999).

3. J. M. Gosline, D. M. Denny, and M. E. DeMond, Nature, 309, 551 (1984).

4. M. A. Becker, D. V. Mahoney, P. G. Lenhert, R. K. Eby, D. Kaplan, and W. W. Adams, "Silk Polymers," ACS Symposium Series 544, Washington, D.C., 1994, p 185.

5. P. M. Cunniff, S. A. Fossey, M. A. Auerbach and J. W. Song, "Silk Polymers", ACS Symposium Series 544, Washington, D.C., 1994, p 234.

6. A. H. Simmons, C. A. Michal, and L. W. Jelinski, Science, 271, 84 (1996).

7. F. Vollrath, Int. J. Biol. Macromol., 24, 81 (1999).

8. S. Osaki and R. Ishikawa, Polym. J., 34, 25 (2002). 
Safety Coefficient of Spider's Lifeline

9. A. Lazaris, S. Arcidiacono, Y. Huang, J. F. Zhou, F. Duguay, N. Chretien, E. A. Welsh, J. W. Soares, and C. N. Karatzas,
Science, 295, 472 (2002).

10. S. Osaki, Acta Arachnologica, 37, 69 (1989). 